\title{
SELEÇÃO DE VANT PARA INSPEÇÕES EM MALHAS RODOVIÁRIAS
}

\author{
Daniel Augusto de Moura Pereira (UFCG) danielmoura@ufcg.edu.br \\ Danillo Marcus Farias Marinho do Monte (UFCG) danilodomonte@gmail.com \\ Marcos dos Santos (IME) marcosdossantos_doutorado_uff@yahoo.com.br \\ Romulo Augustos Ventura da Silva (UFCG) ravs_1987@gmail.com
}

\section{Resumo}

O surgimento de novas tecnologias em diversas áreas tem proporcionado aos profissionais de diversos setores a possibilidade de incremento em seus processos produtivos, sejam eles de bens ou serviços, a fim de obter melhores resultados, redução dos custos operacionais em geral, maior confiabilidade, dentre outros fatores. A identificação dos problemas recorrentes nas malhas rodoviárias do país tem chamado a atenção. Neste sentido, a aplicação de métodos mais modernos tem auxiliado gestores nas suas tomadas de decisão. Sendo assim, a utilização dos VANT para inspeção das malhas rodoviárias, aliado a um sistema de multicritérios e multidecisores (SAPEVO-M), foi utilizado com intuito de determinar qual o melhor equipamento, dentro das categorias de multirotor e asa fixa, a serem utilizados no tipo de inspeção supracitada, levando em consideração os critérios mais relevantes, para o caso em estudo, para realização de inspeções mais eficientes e eficazes.

Palavras-Chaves: SAPEVO-M, VANT, Malhas Rodoviárias, Multicritério.

\section{Introdução}

De acordo com a Confederação Nacional dos Transportes (CNT) (2009), o aumento significativo no fluxo de transportes de passageiros, e de cargas, tem provocado desgastes prematuros das malhas, bem como aumento nos acidentes de trânsito e elevados custos de transporte.

Considerando ainda que o modal rodoviário é o mais utilizado no nosso país e que o Brasil possui uma extensão territorial de proporções continentais, inspecionar e/ou fiscalizar as malhas rodoviárias de nosso país é uma tarefa árdua, custosa para os cofres públicos e que demanda um grande corpo técnico (e infraestrutura) para a realização da mesma. Logo, há necessidade de aplicar novas tecnologias para auxiliar os processos supracitados. 
Tecnologia em evidência, os VANT, conhecidos genericamente por drones, tornaram-se objeto de interesse das mais diversas áreas de conhecimento, consequentemente, promovendo um avanço tecnológico sofisticado e amplamente explorado. Do inglês, denominado como Unmanned Aerial Vehicles/System (UAV/UAS) são definidos como aeronaves projetadas para operar sem piloto a bordo ou autonomamente, e que não tenha caráter recreativo (ANAC, 2017). Nesse sentido, eles podem realizar um papel importante no cenário de inspecionar e/ou fiscalizar malhas rodoviárias com maior índice de eficácia, menor custo e melhorar o processo de tomada de decisão dos gestores. No entanto, nem todos os modelos de VANT disponíveis no mercado servem, a contento, para o propósito comentado anteriormente, seja pela autonomia (da bateria ou de voo) da aeronave, custo, tipo de asa, tecnologia embarcada, entre outras características que, dependendo do estudo a ser realizado, podem ser mais ou menos relevantes.

Portanto, o objetivo deste trabalho é utilizar uma ferramenta de decisão multicritério, o SAPEVO-M, para selecionar qual o melhor VANT a ser utilizado em inspeções de malhas rodoviárias.

\section{Referencial Teórico}

\subsection{Veículo Aéreo Não Tripulado (VANT)}

Para a Associação Brasileira de Aeromodelismo (ABA), o VANT é um veículo capaz de voar na atmosfera, fora do efeito de solo, que foi projetado ou modificado para não receber um piloto humano e que é operado por controle remoto ou autônomo. Medeiros (2007) destaca que os VANT são pequenas aeronaves, sem qualquer tipo de contato físico direto, capazes de executar diversas tarefas, tais como monitoramento, reconhecimento tático, vigilância e mapeamento entre outras. Por sua vez, o Sistema Aéreo Não Tripulado (SANT) significa o conjunto de veículos aéreos não tripulados, seus controles de voo e seu sistema de operação, isto é, a união de todas as atividades que estão interligadas no plano de voo (RASI, 2008). De acordo com Neris (2001), os VANT têm como componente principal um sistema de controle capaz de manter a aeronave estabilizada e de executar manobras que a conduza através de uma rota e missão selecionada. A Figura 1 ilustra os componentes principais de um VANT. 


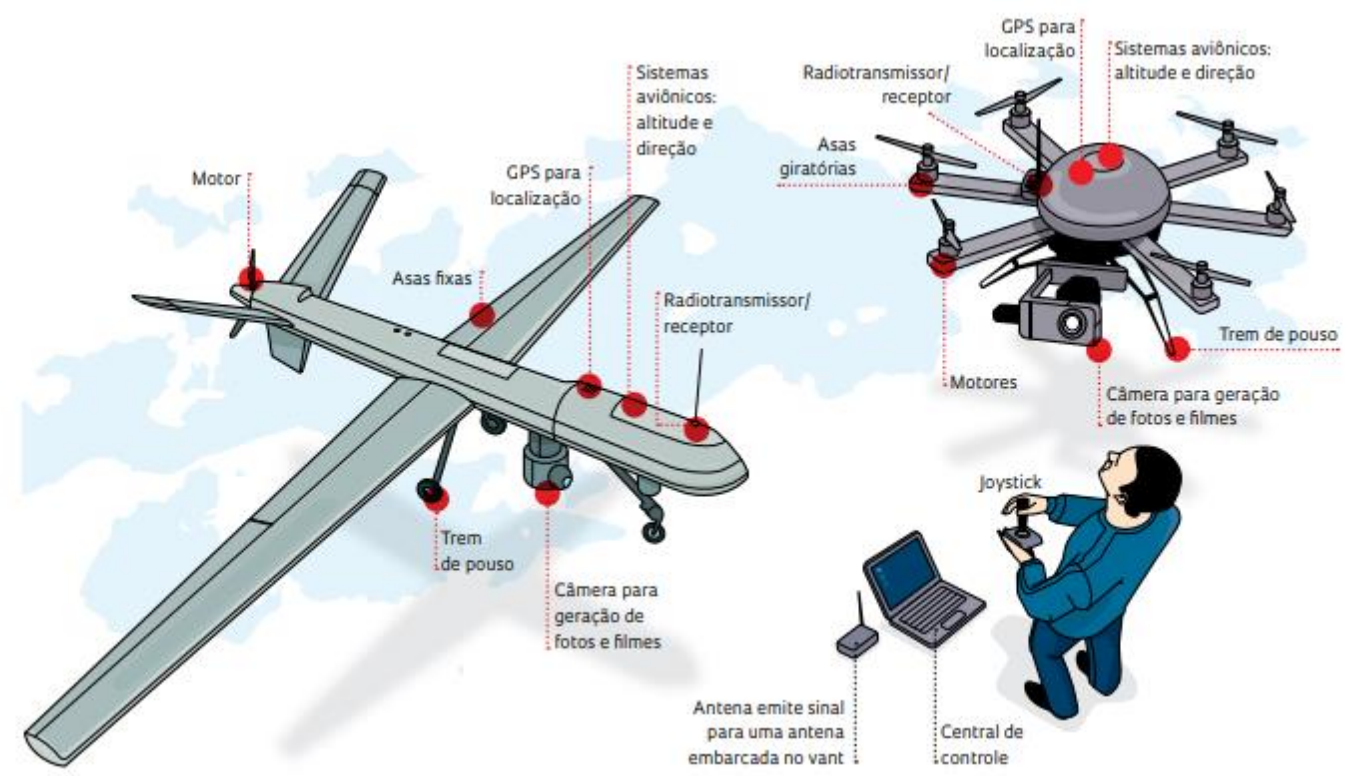

Fonte: Adaptado de Andrade (2013)

\subsection{Inspeção com VANT}

A evolução das plataformas VANT tem possibilitado a utilização desta tecnologia em diversas aplicações, tais como: agricultura e pecuária, vigilância e controle de tráfego, monitoramento de situações de emergência, como incêndios e desastres naturais, campanhas publicitárias, mercado imobiliário, além dos diferentes domínios da engenharia civil a qual dar-se-á maior ênfase devido a ser parte do objeto de estudo o envolvimento com malhas rodoviárias federais . Tal potencial de uso está diretamente relacionado ao baixo custo, à alta mobilidade, à segurança oferecida e à velocidade de aquisição e transferência de dados, como imagens e vídeos (MORGENTHAL; HALLERMANN, 2014; KIM; IRIZARRY, 2015). Além disso, o VANT pode ser utilizado em situações em que uma inspeção tripulada não seja possível ou até mesmo a inspeção in loco também não possa ser realizada (MORGENTHAL; HALLERMANN, 2014).

Na engenharia civil, os estudos são recentes, tendo como destaque: as áreas de infraestrutura de transportes, para monitoramento e manutenção de pavimentos, rodovias (ZHANG, 2008; THEMISTOCLEOUS et al., 2014); inspeção e monitoramento de pontes (METNI; HAMEL, 2007; MORGENTHAL; HALLERMANN, 2014). 
O VANT tem se mostrado uma excelente ferramenta de obtenção de imagens com resoluções melhores, a baixo custo e alta resolução temporal. Essas características têm popularizado seu uso, principalmente para atualização e confecção de mapas, em áreas afastadas ou próximas onde o uso da fotogrametria convencional torna a execução do trabalho economicamente inviável. Sua utilização vem ganhando mercado em razão da sua agilidade na tomada de imagens e no processamento destas, gerando resultados em tempo real. Se comparado a outros sensores disponíveis, os VANTs se destacam devido à sua resolução espacial, liberdade ao operador do equipamento para a obtenção de imagens em diferentes momentos, facilidade no desenvolvimento de planos de voos, além da grande possibilidade de se estruturar uma base com imagens multi temporais (BRAZ, et al., 2015).

Diante da necessidade de se monitorar áreas e realizar mapeamentos em grande escala, a aplicação de VANT vem se despontando no mercado de mapeamentos digitais, e trazendo características, como ganho na resolução espacial e temporal (ALONÇO et al. 2005).

\subsection{Método Ordinal SAPEVO-M}

De acordo com Santos et al. (2017), os métodos que empregam múltiplos critérios em auxílio à tomada de decisão têm um caráter científico e, concomitantemente, subjetivo, trazendo consigo a capacidade de combinar, de maneira ampla, as características consideradas importantes, inclusive as não quantitativas, com o objetivo de possibilitar a transparência e sistematização do processo referente aos problemas de decisão (Santos et al. 2017). O método SAPEVO-M, como nova versão do método ordinal SAPEVO (Gomes et al. 1997) original, possibilita a utilização de apenas um decisor. Além disso, introduziu um processo de normalização das matrizes de avaliação, incrementando a consistência do modelo. Evitando a necessidade de uma pré ordem dos critérios, e a mesma ser obrigatoriamente de consenso dos decisores.

Basicamente, o método consiste em dois processos. Preliminarmente, deve ser realizada a transformação da preferência ordinal entre critérios, a ser expressa por um vetor representando os pesos dos critérios. Em seguida, é feita a transformação ordinal da preferência entre alternativas dentro de um determinado conjunto de critérios, expressa por uma matriz. Uma série de comparações pareadas entre as opções quer seja critérios ou alternativas dentro de um determinado critério, denotam as informações de preferência individual de cada decisor (TEIXERA, SANTOS e GOMES, 2019) 
Sejam ci e cj dois critérios dentro de um conjunto de critérios $\mathrm{C}=\{\mathrm{c} 1, \mathrm{c} 2, \ldots \mathrm{ci}, \ldots, \mathrm{cj}, \ldots\}$, o grau de preferência entre eles é dado por $\delta$ ci cj, no qual:

$\delta$ ci cj $=1 \leftrightarrow \mathrm{ci} \cong \mathrm{cj}$, isto é, ci é tão importante quanto cj;

$\delta$ ci cj $>1 \leftrightarrow c i>c j$, isto é, ci é mais importante que cj ;

e $\delta$ ci cj $<1 \leftrightarrow$ ci $<$ cj, isto é, ci é menos importante que cj.

Uma escala de sete pontos expressa a relação entre as alternativas, na qual são mensuradas, relativamente, a importância entre cada opção. A partir da avaliação entre alternativas, é obtido uma matriz com a representação numérica correspondente. A relação entre a escala de preferência e o valor numérico é expressa na Tabela 1.

Tabela 1 - Relação entre escala de preferência e o valor numérico

\begin{tabular}{|c|c|}
\hline Preferência & Escala numérica \\
\hline$<<\ll 1$ & -3 \\
\hline$<\ll 1$ & -2 \\
\hline$« 1$ & -1 \\
\hline 1 & 0 \\
\hline$» 1$ & 1 \\
\hline$>» 1$ & 2 \\
\hline$>>» 1$ & 3 \\
\hline
\end{tabular}

Fonte: Adaptado de Teixera, Santos e Gomes (2019)

Seja D um conjunto de atores que decidem Decision Makers $(\mathrm{DM}), \mathrm{D}=\{\mathrm{DM} 1, \mathrm{DM} 2, \ldots$, $\mathrm{DMk}, \ldots, \mathrm{DMn}, \ldots\}$ que expressam suas opiniões sobre os critérios em ordem de preferência. Para um agente decisor DMk, estas informações geram uma matriz de avaliação MDMk. A relação entre as duas escalas da tabela 1 permite a transformação da matriz MDMk $=[\delta \mathrm{ci} \mathrm{cj}]$ em um vetor coluna $[\mathrm{Vi}]$ de tal forma que:

$\mathrm{Vi}=$, observando-se a escala 2 da tabela 1 para $\mathrm{i}=\{1, \ldots, \mathrm{m}\}$ e $\mathrm{k}=\{1, \ldots, \mathrm{n}\}$. O vetor $\mathrm{V}$ representa as preferências entre critérios de cada DM.

Este método prevê, também, terminado o processo de integração da matriz, a normalização do vetor resultante. Esta normalização garante a geração de valores não negativos, por meio da equação $v=[($ aij - Mín aij $) \div($ Máx aij - Mín aij) $]$. Durante a normalização dos critérios, os menores valores, dos pesos dos critérios, assumem valor zero, sendo substituídos por um valor arbitrado de $1 \%$ do valor imediatamente superior; este procedimento deve-se ao fato de 
não fazer sentido que um determinado critério possua peso nulo. A escolha da fórmula de normalização foi feita após uma análise dos quatro métodos matemáticos de normalização (TEIXERA, SANTOS e GOMES, 2019).

De maneira análoga ao procedimento para obtenção do vetor peso dos critérios, dado um conjunto de alternativas, estas são comparadas par a par, à luz de cada critério. Desta forma, transforma-se a matriz $\mathrm{E}=\{$ ei,k,l] em um vetor coluna $\mathrm{Ai}$, onde ei,k,l é o valor da comparação pareada da alternativa ak com a alternativa al dentro do critério I, para cada agente decisor DM. Logo, obtém-se o vetor coluna A, onde $\mathrm{A}=\sum \mathrm{ADM}$ da matriz $\mathrm{M}(\mathrm{n} \mathrm{x} \mathrm{m})$ ). Alterando-se o critério I na qual as alternativas foram avaliadas, procede-se novamente a comparação pareada e a integração do vetor, de modo que a matriz $\mathrm{M}$ seja toda preenchida. (TEIXERA, SANTOS e GOMES, 2019)

O resultado da preferência entre as alternativas é expresso pelo vetor resultante da multiplicação matricial entre o vetor peso dos critérios $\mathrm{V}$ e a matriz de avaliação das alternativas M. As alternativas são, então, ordenadas em ordem decrescente dos valores numéricos obtidos, gerando-se o ranking desejado, indicando, portanto, a melhor alternativa.

\section{Metodologia}

Trata-se de um trabalho descritivo, exploratório e quali-quantitativo. O intervalo do estudo aconteceu no último semestre de 2019, em malhas rodoviárias do Estado da Paraíba. O esquema metodológico utilizado nesta pesquisa pode ser visualizado na Figura 2.

O procedimento metodológico utilizado neste estudo foi dividido em 5 fases. Inicialmente, foram realizadas visitas in loco em duas malhas rodoviárias de fluxo intenso do Estado da Paraíba, método de observação direta e registros fotográficos para levantamento e identificação dos principais problemas lá existentes. Em seguida, definiu-se o problema a ser investigado nesta pesquisa baseado em duas perspectivas: tempo demandado para realizar as inspeções, grau de assertividade da inspeção e custo. A posteriori, foi realizada uma entrevista não estruturada com um gestor do Departamento Nacional de Infraestrutra de Transporte (DNIT) e com um piloto de VANT, e o problema foi analisado da perspectiva do sistema produtivo em estudo. Neste sentido, foram definidos os requisitos necessários que os VANT deveriam atender para suprir as demandas relacionadas as inspeções e/ou fiscalizações nas malhas rodoviárias. 
Figura 2 - Esquema metodológico utilizado na pesquisa.

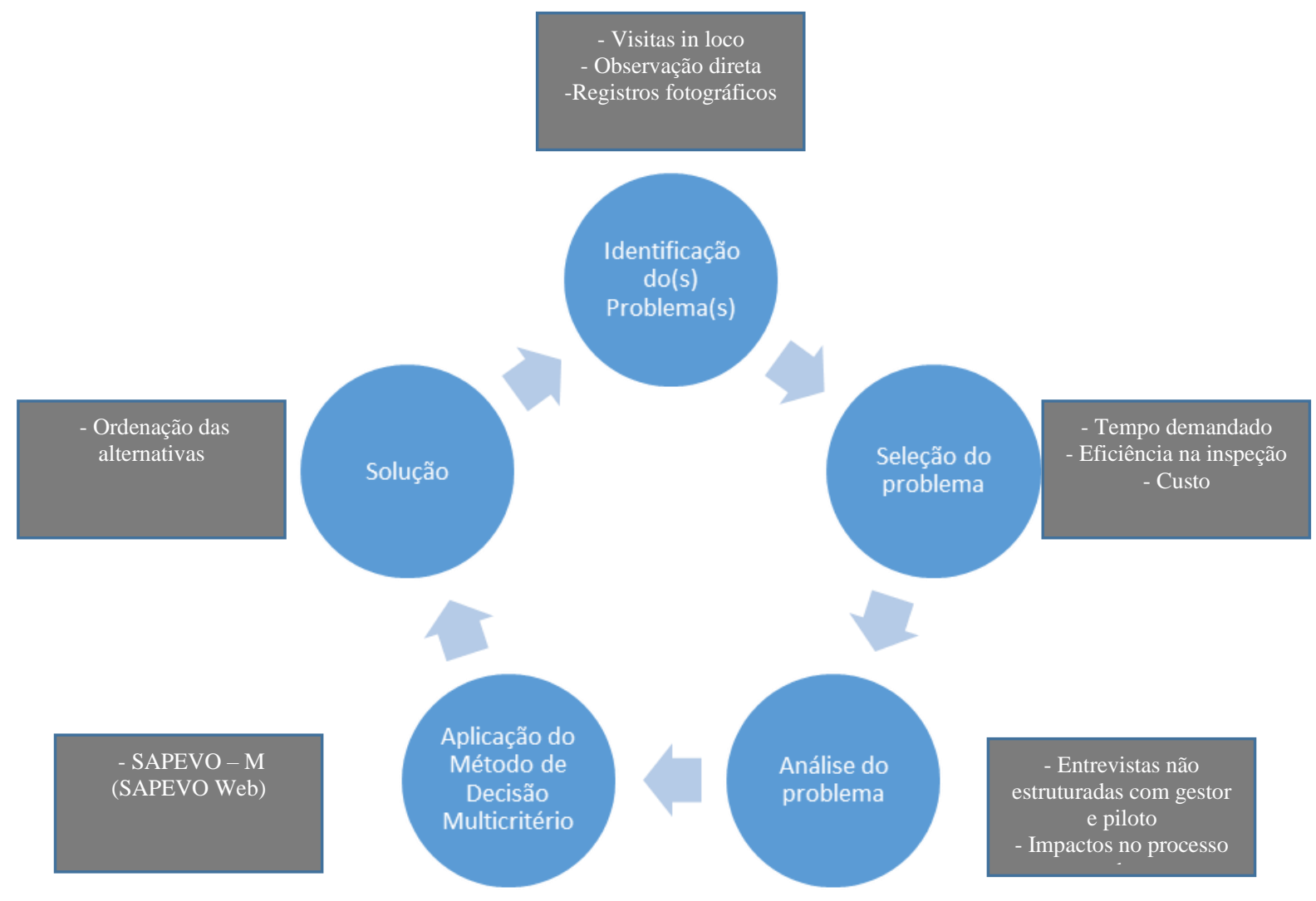

Fonte: Autores (2020)

Os critérios necessários para avaliar quais equipamentos podem fornecer maior custo/benefício foram: marca/modelo, alcance máximo, autonomia, câmera, manutenção, preço, peso (asas fixas) e velocidade. Os VANT em questão estão expostos na Tabela 2 subdivididos entre multirotor e asa fixa. 
Tabela 2 - VANT Avaliados

\begin{tabular}{cc}
\hline Multirotor & Asa Fixa \\
\hline DJI Mavic 2 & Horus Verok \\
DJI Mavic Air & Horus Maptor HS \\
DJI Phantom 4 Pro & Batmap I \\
DJI Phantom 4 Adv & Batmap II \\
DJI Matrice 200 & \\
Parrot Anafi Extend & \\
DJI Inspire 2 & \\
Nuvem UVA Spectral & \\
\hline
\end{tabular}

Fonte: Os Autores (2020)

O quarto passo foi aplicar um Modelo de Apoio à Decisão Multicritério. O modelo escolhido para a solução do problema foi o SAPEVO-M, juntamente com a ferramenta SAPEVO Web. Basicamente, o método consiste em dois processos. Preliminarmente, deve ser realizada a transformação da preferência ordinal entre critérios, a ser expressa por um vetor representando os pesos dos critérios. Em seguida, é feita a transformação ordinal da preferência entre alternativas dentro de um determinado conjunto de critérios, expressa por uma matriz. Uma série de comparações pareadas entre as opções quer seja critérios ou alternativas dentro de um determinado critério, denotam as informações de preferência individual de cada decisor (Gomes et al. 1997). A ferramenta supracitada para tratamento e análise dos dados pode ser acessada através do endereço: http://www.SAPEVOweb.com.

Por fim, após a aplicação do método SAPEVO-M, o output gerado foi o ordenamento das alternativas do melhor para o pior fornecedor, segundo os requisitos determinados pelos gestores e pelos pilotos.

\section{Resultados}


A plataforma SAPEVO Web utiliza uma escala que varia de -3 a +3 , de acordo com a Tabela 1, a qual se classifica de absolutamente pior a absolutamente melhor, além de fornecer a possibilidade de tomada de decisão por multi decisores, ou seja, para avaliação dos modelos tem que haver dois ou mais decisores envolvidos no processo de tomada de decisão.

Sendo assim, o sistema foi avaliado por dois decisores aos quais tiveram que classificar os critérios de acordo com a Tabela 3.

Tabela 3 - Escala de critérios

\begin{tabular}{cc}
\hline Expressão Linguística Correspondente & Escala \\
\hline Absolutamente Pior & -3 \\
Muito Pior & -2 \\
Pior & -1 \\
Equivalente & 0 \\
Melhor & 1 \\
Muito Melhor & 2 \\
Absolutamente Melhor & 3 \\
\hline
\end{tabular}

Fonte: Adaptado de Texeira, Santos e Gomes (2019)

Após o decisor 1 e o decisor 2 expressarem as suas opiniões, e após a comparação paritária entre os critérios de ambos, o SAPEVO classificou, no modelo multirotor, o Matrice 200 como sendo a melhor opção. Os critérios que obtiveram maior peso foram: alcance, autonomia e preço. Veja na Figura 3.

Figura 3 - Esquema metodológico utilizado na pesquisa. 


\section{Resultado | Projeto D 1 \\ Pesos}

Critério - Alcance (km) - 1.8461538461538463
Critério - Autonomia (min) - 1.8333333333333335
Critério - Câmera - 0.8846153846153846
Critério - Manutenção - 0.008846153846153847
Critério - Velocidade - 1.5192307692307692
Critério - Preço R\$ - 1.608974358974359

Ordenação

10 -- Matrice 200 -- 9.819179905293185

$2^{\circ}$-- Phantom 4 Adv -- 8.76770847977257

$3^{\circ}$-- Phantom 4 Pro -- 8.488915684416426

$4^{\circ}$-- Mavic 2 -- 8.463405739233403

$5^{\circ}$-- Spectral -- 7.991973014165321

$6^{\circ}--$ Inspire $2-7.354409869485935$

70 -- Parrot ANAFI -- 4.973876252634406

$8^{\circ}$-- Mavic Air -- 3.431911101718794

Fonte: Os Autores (2020)

Assim como no resultado do Projeto D1 (Figura 3), o Projeto D2 demonstrou qual a melhor opção custo/benefício, e os critérios que obtiveram maior peso também foram alcance, autonomia e preço, indicando o HORUS Verok como a melhor opção de acordo com a Figura 4.

Figura 4 - Resultado Projeto D2. 


\section{Resultado | Projeto Projeto D 2}

Pesos

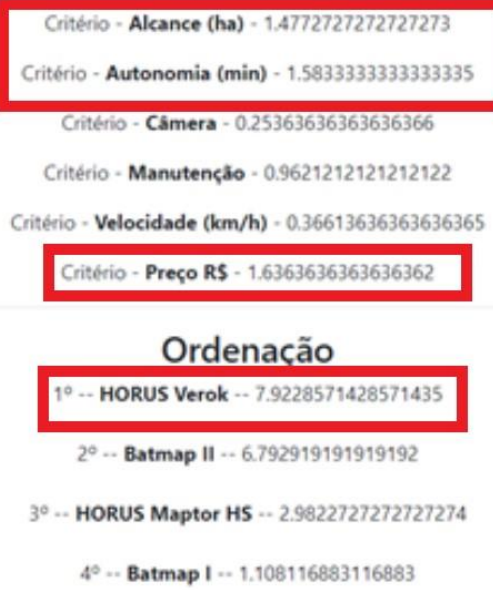

Fonte: Os Autores (2020)

As combinações paritárias de ambos os decisores corroboraram para resultado satisfatório ao qual indicou o equipamento que melhor se enquadra na inspeção de pavimentos rodoviários federais, fatos estes que foram elucidados no início do artigo. Ainda assim, alguns fatores necessitam de analises minuciosas.

Fatores como alcance e autonomia possuem relação direta, tendo em vista que o mapeamento rodoviário possui melhor eficácia/eficiência se for realizado de forma linear, portanto, o equipamento que oferecer a melhor relação Alcance x Autonomia será o mais indicado. De fato, o VANT multirotor eleito como melhor opção pelo SAPEVO oferece a melhor relação supracitada, como pode ser visto no Gráfico 1. 
Gráfico 1 - Comparativo Alcance x Autonomia (Multirotores)

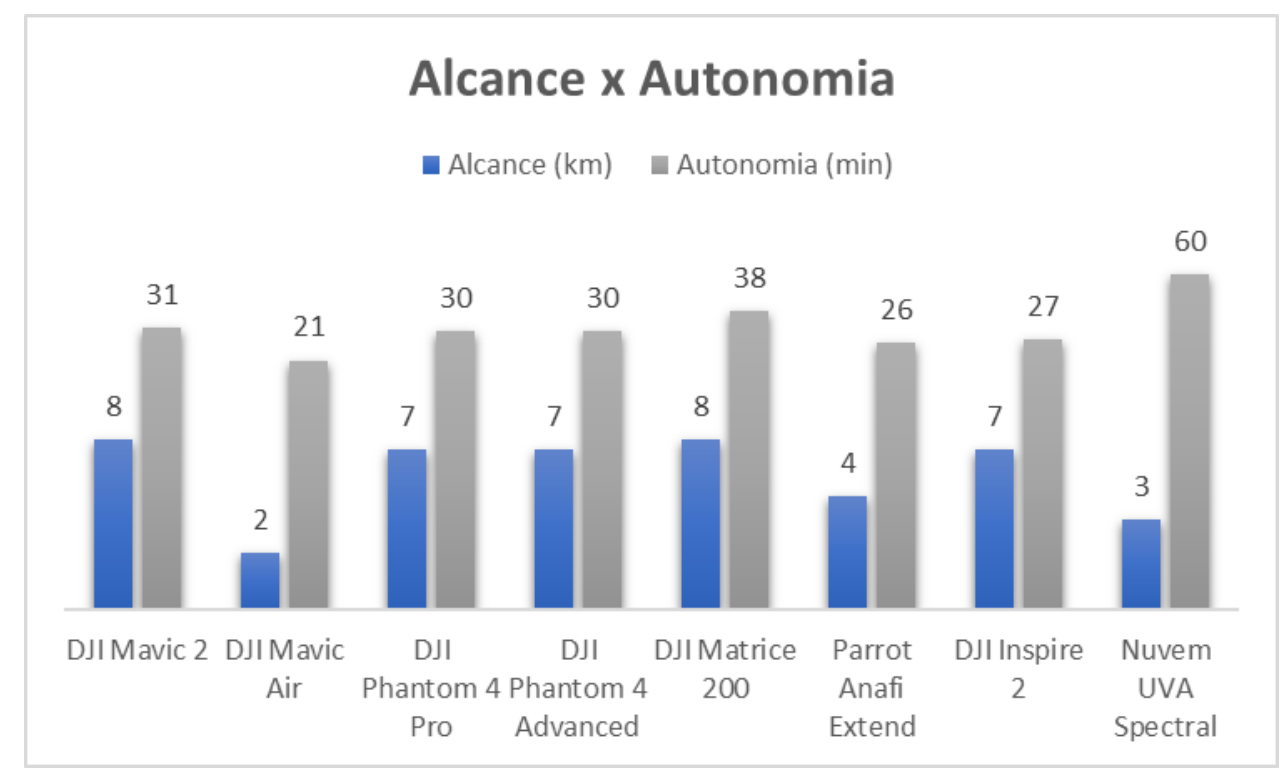

Fonte: Os Autores (2020)

A mesma observação também é válida para os VANT de asas fixas, sendo que neste caso o critério preço teve um peso bastante relevante, sendo este o critério de "desempate" entre o $1^{\circ}$ e o segundo $2^{\circ}$ colocado. O Gráfico 2 mostra a relação Alcance x Autonomia dos VANT de asas fixas.

Gráfico 2 - Comparativo Alcance x Autonomia (Asas fixas)

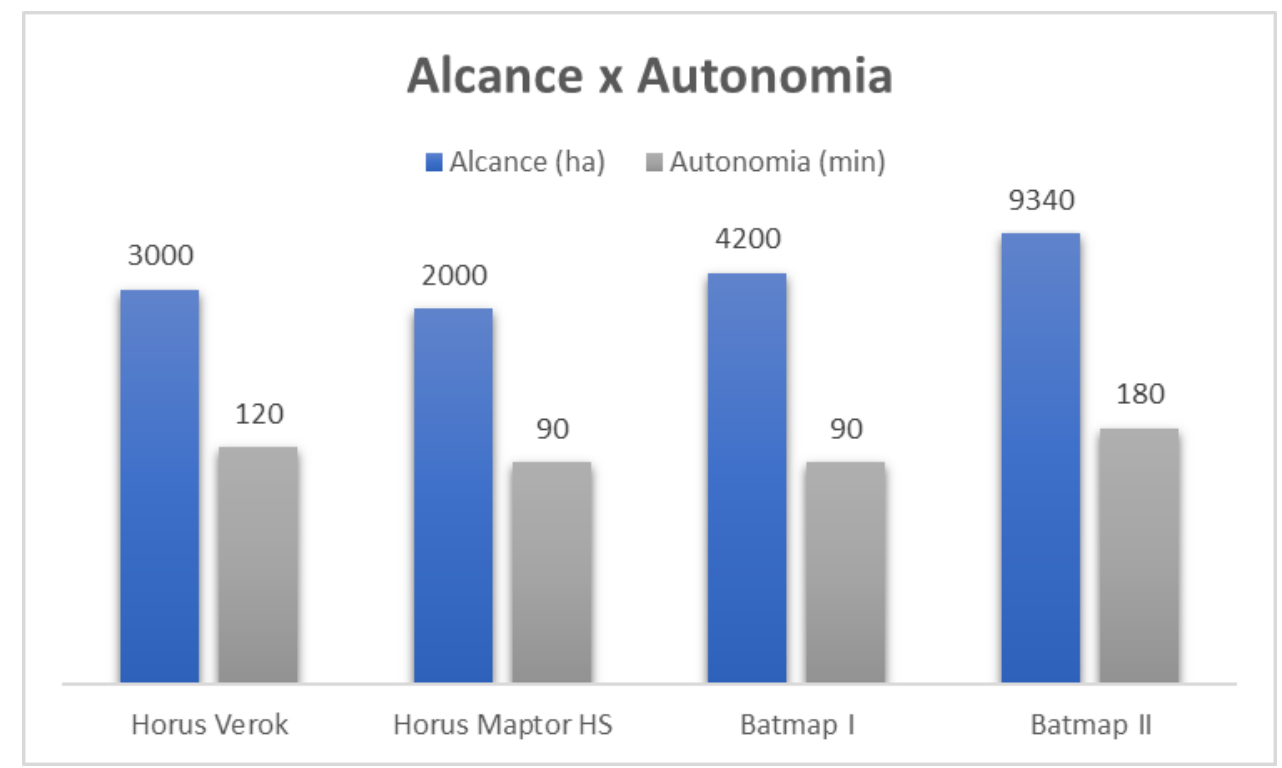

Fonte: Os Autores (2020)

Um terceiro fator que mostrou fator relevante em ambos processos decisórios foi o preço. No caso dos multirotores o preço teve o terceiro maior peso sendo os fatores de maior decisão os que oferecem critérios mais técnicos, se sobressaindo alcance e autonomia. O Gráfico 3 demonstra o preço dos multirotores. 
Gráfico 3 - Critério de decisão (Preço)

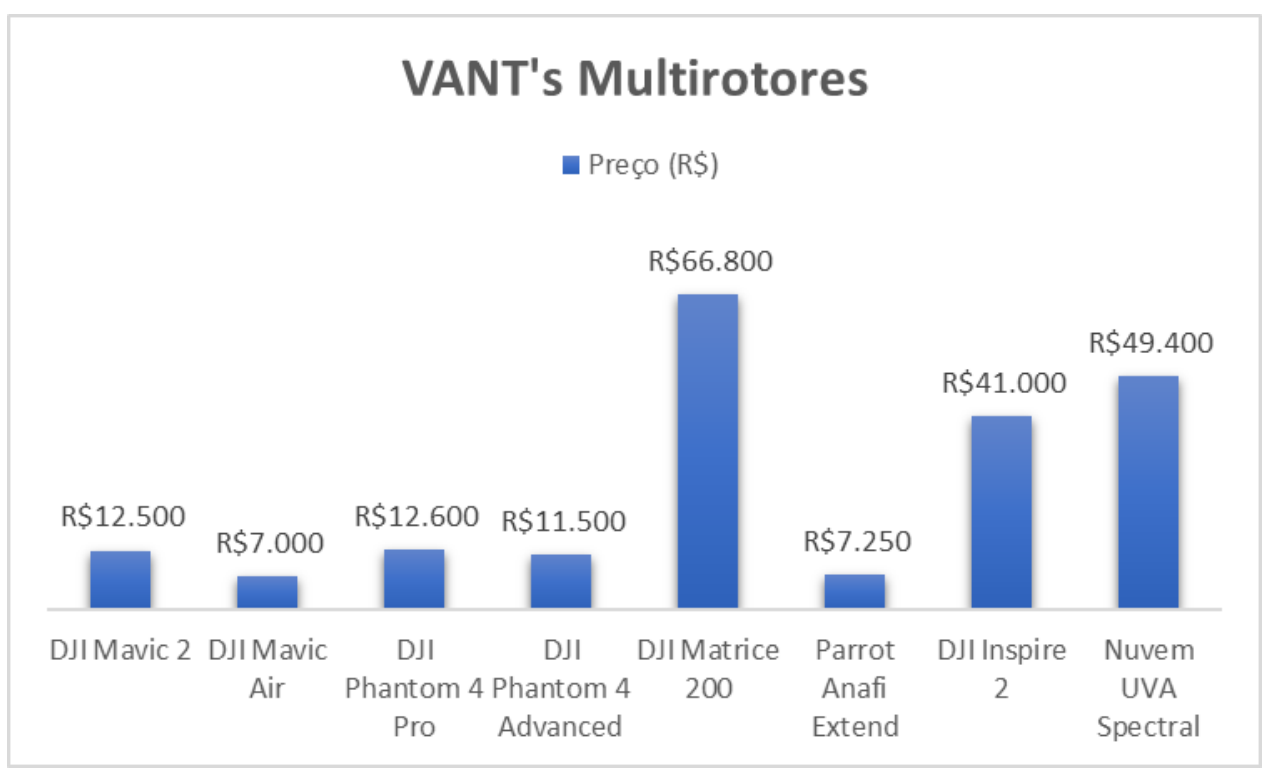

Fonte: Os Autores (2020)

É um tanto quanto controverso o DJI Matrice 200 apresentando-se como a melhor escolha. Uma possível explicação para este caso, é que no processo multidecisor, ambos os decisores atribuíram valores paritários que levaram em consideração os requisitos técnicos além dos critérios que advém do conhecimento empírico, sendo levado em consideração fatores como: sustentação, estabilidade e confiabilidade durante o voo. Os outros equipamentos dificilmente atingem os números prometidos pelo manual do fabricante, principalmente, quando se diz respeito a autonomia de voo. Vale ressaltar que fatores externos (clima, deslocamento de ar e altitude de voo) influenciam diretamente no critério autonomia.

Já nos VANT de asa fixa, o critério preço obteve maior peso tendo em vista de que todos os modelos em avaliação possuem configurações e estruturas semelhantes. Portanto, o Horus Verok apresentou melhor custo benefício, como pode ser visto no Gráfico 4. 
Gráfico 4 - Critério de decisão (Preço)

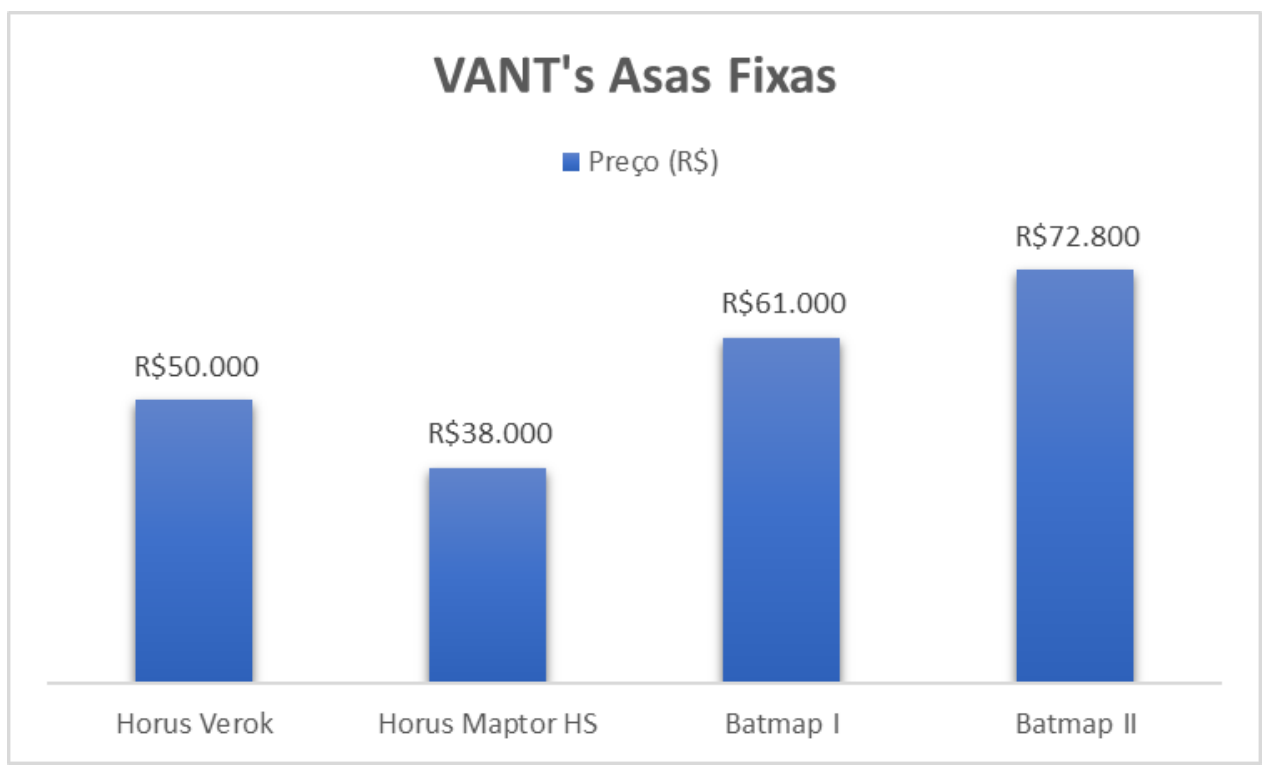

Fonte: Os Autores (2020)

Os resultados mostrados nos Gráfico 3 e 4 apontaram, entre os modelos, multirotor e asa fixa, os mais adequados, em cada categoria, para inspeção de malhas rodoviárias, tendo em vista os critérios que foram levados em consideração nesta pesquisa. Nota-se também, que os VANT's mais robustos se sobressaem com relação aos demais, mesmo apresentando preços muito além dos seus concorrentes.

Com relação ao critério manutenção, foi levado em consideração a facilidade, preço e acessibilidade da mesma, tendo em vista que a maioria dos VANT multirotores não possuem fábrica no Brasil. Diferentemente, os asas fixas são fabricados no Brasil, fator este que pode abrir uma grande vantagem entre os multirotores e asas fixas.

\section{Considerações Finais}

Esta pesquisa teve como objetivo utilizar uma ferramenta de decisão multicritério, o SAPEVO-M, para selecionar qual o melhor VANT a ser utilizado em inspeções de malhas rodoviárias. Para tanto, os VANT foram divididos em duas categorias principais: multirotor e asa fixa.

Após a aplicação do método de decisão supracitado, os resultados indicaram que para a categoria de multirotor, o melhor VANT é o DJI Matrice 200. Já para os VANT de asa fixa, a melhor escolha foi o Horus Verok.

Por fim, pode-se dizer que há a possibilidade de se fazer os mapeamentos com os VANT que ocuparam as ultimas posições. Todos eles possuem recursos para realização de inspeção em 
malhas rodoviárias. A seleção deles irá depender da extensão do trecho avaliado e finalidade do projeto a ser feito. De qualquer forma, quanto mais confiável e adequado for o equipamento, mais eficiente e eficaz se tornará o mapeamento e o produto final.

\section{REFERÊNCIAS}

AGÊNCIA NACIONAL DE AVIAÇÃO CIVIL (ANAC). Regulamento Brasileiro da Aviação Civil Especial (RBAC-E $\mathbf{n}^{\mathbf{0}}$ 94).<https://www.anac.gov.br/assuntos/legislacao/legislacao-1/rbha-e-rbac/rbac/rbac-e-94> Acesso em: 10 jan.2020

CNT - Confederação Nacional dos Transportes. Pesquisa Rodoviária, 2009.

CNT - Confederação Nacional dos Transportes. Pesquisa Rodoviária, 2019.

GOMES, L. F. A. M., e GOMES, C. F. S. Princípios e métodos para a tomada de decisão: Enfoque multicritério (6a ed.). São Paulo: Atlas. (2019).

SANTOS, M. GOMES, C. F. S. OLIVEIRA, A. S. COSTA, H. G. Uma abordagem multicritério para seleção de um navio de guerra de médio porte a ser construído no Brasil. In: XLVIII Simpósio Brasileiro de Pesquisa Operacional, 2016, Vitória, Espírito Santo. p.507 a 518. (2016)

TEIXEIRA, L. F. H. S. B., SANTOS, M. e GOMES, C. F. S. SAPEVOWeb Software (v.1). (2018). Disponível em: Acesso em: 18/01/ 2020.

MEDEIROS, F. A. Desenvolvimento de um veículo aéreo não tripulado para aplicação em agricultura de precisão, 2007, 102f. Dissertação (Mestrado em Engenharia Agrícola) - Universidade Federal de Santa Maria, Santa Maria - RS.

MORGENTHAL, G.; HALLERMANN, N. Quality assessment of Unmanned Aerial Vehicle (UAV) based visual inspection of structures, Advances in Structural Engineering, v. 17, n. 3, 2014

NERIS, L.O. Um piloto automático para as aeronaves do projeto ARARA, 2001. 102f. Dissertação (Mestrado em Ciência da Computação), Universidade de São Paulo, São Carlos.

RASI, J. R. Desenvolvimento de um veículo aéreo não tripulado para aplicação em pulverização agrícola, 2008. Dissertação Pós-Graduação Faculdade de Agronomia Eliseu Maciel. Pelotas, RS, Brasil.

ZHANG, C. Development of a UAV-based remote sensing system for unpaved road condition assessment. In: American Society for Photogrammetry and Remote Sensing Annual Conference, Portland, Oregon, 2008.

NEVES, Roberta Braga; PEREIRA, Valdecy and COSTA, Helder Gomes. Auxílio multicritério à decisão aplicado ao planejamento e gestão na indústria de petróleo e gás. Produção. 2015, vol.25, n.1, pp.43-53. Epub Sep 03, 2013.. 